Rob Gonsalves*, Ramin Jamshidi, Dorothee Newbern, Gabriel Shaibi, Micah Olson, Reeti Chawla, Sharon H. Gnagi and Oliver J. Oatman

\title{
A quality improvement project for managing hypocalcemia after pediatric total thyroidectomy
}

https://doi.org/10.1515/jpem-2020-0376

Received June 24, 2020; accepted September 21, 2020;

published online October 7, 2020

\section{Abstract}

Objectives: Hypocalcemia following total thyroidectomy (TT) is relatively common. It may result in significant morbidity, prolonged hospital stay, and increased costs. Treatment with intravenous (IV) calcium gluconate may also carry significant risks. In pediatrics, management consensus guidelines are lacking.

Methods: At Phoenix Children's Hospital, a team of pediatric endocrinologists, surgeons and otolaryngologists developed a clinical pathway for patients undergoing TT. It was a Quality Improvement (QI) project with the primary aim of decreasing IV calcium gluconate use from a baseline of $68 \%$ to less than $40 \%$ over 15 months. Secondary aims included reducing hypocalcemia and length of hospitalization. Interventions included sending weekly pathway reminder emails, starting pre-operative calcium, and pathway implementation into the electronic health record. Results: Twenty-seven patients underwent TT over 15 months. IV calcium gluconate use dropped to $48 \%$. Hypocalcemia and length of hospitalization were $96 \%$ and $52.7 \mathrm{~h}$ (range 21.1-115.7) respectively. Pathway adherence improved after targeted interventions. Eleven (73\%) of the 15 patients whose post-operative parathyroid hormone (PTH) nadir was below $15 \mathrm{pg} / \mathrm{mL}$ required IV calcium gluconate vs. two (17\%) out of 12 with levels above this threshold.

\footnotetext{
*Corresponding author: Rob Gonsalves, DO, Phoenix Children's Hospital, Division of Endocrinology and Diabetes, 1919 E Thomas Road, Phoenix, 85016 AZ, USA, Phone: 602933 0179,

E-mail: rgonsalves@phoenixchildrens.com

Ramin Jamshidi, Phoenix Children's Hospital, Division of Pediatric Surgery, Phoenix, AZ, USA

Dorothee Newbern, Micah Olson, Reeti Chawla and Oliver J. Oatman, Phoenix Children's Hospital, Division of Endocrinology and Diabetes, Phoenix, AZ, USA

Gabriel Shaibi, Arizona State University College of Nursing and Health Innovation, Center for Health Promotion and Disease Prevention, Phoenix, AZ, USA

Sharon H. Gnagi, Phoenix Children's Hospital, Department of Otolaryngology, Phoenix, AZ, USA
}

Conclusions: Standardizing care allowed for objective outcome analysis. We learned that post-operative serum PTH level was the main risk factor for requiring IV calcium gluconate. Implementing the pathway as a QI project allows for revisions based on outcomes, ultimately resulting in a pathway that best utilizes our infrastructure to optimize care. Other pediatric institutions may face similar challenges and can potentially learn from our experience.

Keywords: hypocalcemia; quality improvement; total thyroidectomy.

\section{Introduction}

Total thyroidectomy (TT) is a relatively common pediatric surgical procedure, typically performed for Graves' disease or thyroid neoplasia. Hypocalcemia is a common occurrence following pediatric total thyroidectomy, with incidence ranging from 13 to $73 \%$ [1-3]. It is caused by hypoparathyroidism due to manipulation, devascularization, or removal of parathyroid glands during surgery. This hypoparathyroidism is usually temporary, however in rare cases can be permanent. Hypocalcemia can result in significant morbidity, including muscle cramps and spasms, seizures, arrhythmia, and even death. Treatment of severe hypocalcemia with intravenous (IV) calcium gluconate is not without risk, as there is the possibility of extravasation with resultant tissue necrosis. This has the potential to prolong hospital stay and increase costs for both the hospital and family.

Management of hypocalcemia after total thyroidectomy can be challenging, and no consensus guidelines have been developed. There is a relative paucity of literature pertaining to management of post-operative hypocalcemia following TT in pediatric patients, though some studies have emerged in recent years $[1,4,5]$.

Risk factors for post-operative hypocalcemia in children include younger age, hyperthyroidism, and concomitant neck dissection [6, 7], the latter of which is more often required for pediatric patients given the propensity of thyroid cancer to metastasize to the lymph nodes in this age group [8]. Post-operative parathyroid hormone (PTH) levels are a well-studied predictor of hypocalcemia after TT 
in adults [9], however there are limited studies on the use of PTH in stratifying risk of hypocalcemia in pediatric patients $[5,10]$.

Hypoparathyroidism and hypocalcemia after TT is a dynamic process that requires close monitoring along with early medical intervention if needed. These patients are often managed by multiple providers across subspecialties. Clear communication and coordinated management strategies are imperative and could be aided by clinical pathways.

One of the strategic patient quality and safety goals at our institution is to standardize and optimize care through the design and implementation of clinical pathways. We therefore aimed to develop a clinical pathway for managing hypocalcemia after total thyroidectomy in a standardized manner. The goals were to reduce the use of IV calcium gluconate, incidence of hypocalcemia, and length of stay (LOS). The pathway was carried out in the context of a quality improvement (QI) project using the Plan-DoStudy-Act (PDSA) model.

\section{Materials and methods}

Baseline measurements: To understand the management practices and variability, a retrospective chart review was performed on patients who underwent TT at our institution in 2017. Data extracted included rates of hypocalcemia, medications used for the treatment of hypocalcemia, labs used for hypocalcemia monitoring, and LOS. The chart review identified 19 total thyroidectomies performed, with 18 (95\%) experiencing biochemical hypocalcemia defined by a serum calcium of $<8.0 \mathrm{mg} / \mathrm{dL}$ [2.0 mmol/L]. Thirteen patients $(68 \%)$ required IV calcium gluconate management. There was heterogeneity pertaining to laboratory monitoring, specifically with regard to whether or not pre-operative calcium and PTH were obtained, timing of initial post-operative labs, and whether serum or ionized calcium was used to monitor calcium levels. The average LOS was 52.9 h (range 20.6-189.3) with a median LOS of $44.7 \mathrm{~h}$.

Pathway development and description: To supplement institutional data, the Pediatric Health Information System (PHIS) database, a comparative pediatric database with clinical and resource utilization data from over 52 children's hospitals, was queried to identify high volume pediatric centers performing total thyroidectomies. We subsequently reached out to the highest volume institutions to determine if they use management pathways, and if so, compare the clinical components of those pathways. A literature review pertaining to calcium monitoring and management after TT was also conducted. The data from the chart review, PHIS query, and literature review were used to engage providers from our institution's Endocrinology, Pediatric Surgery, and Otolaryngology services to discuss the development of a clinical pathway to address hypocalcemia management following TT. Through an iterative process, we developed a clinical pathway for monitoring and managing hypocalcemia after TT (Figure 1).

The pathway helps guide care for all patients undergoing TT at our institution regardless of the indication. TTs are performed by a selected group of surgeons from the Pediatric Surgery and Otolaryngology services. Pre-operative labs were standardized to include serum calcium, albumin, and PTH. Thyroid function was optimized pre-operatively for patients with Graves' disease. For severe cases, this required several days of potassium iodide drops and glucocorticoids in addition to methimazole.

In its current iteration, the pathway instructs to start all patients on pre-operative calcium carbonate. This is either continued, or increased based on the calcium and PTH thresholds outlined in the pathway. Calcium and PTH are then obtained in the post-anesthesia care unit, with subsequent calcium levels drawn every $6 \mathrm{~h}$, and PTH levels drawn every morning. Oral calcitriol is started for any corrected calcium less than $8.0 \mathrm{mg} / \mathrm{dL}$ [2.0 mmol/L] or if there is a drop in PTH greater than $50 \%$ from pre-operative baseline. IV calcium gluconate is given for any calcium less than $7.0 \mathrm{mg} / \mathrm{dL}$ [1.75 mmol/L] or for symptoms of severe hypocalcemia such as seizure. These values were determined based on clinical experience and the expert opinion of our group. If IV calcium gluconate is given, an electrocardiogram is obtained and calcium level is repeated in $1-2 \mathrm{~h}$ to determine subsequent management. Patients are considered clear for discharge when serum calcium is stable (not decreasing) without the need for IV calcium gluconate in the previous $24 \mathrm{~h}$. Post-operative calcium, albumin, and PTH are obtained 3-5 days after discharge, and medications are subsequently adjusted by either the on-call endocrine team or the patient's primary endocrinologist.

The pathway was implemented in the context of a QI project with the primary aim to decrease the rate of IV calcium gluconate use from $68 \%$ observed in the chart review to less than $40 \%$ over a time period of 15 months. Secondary aims included reducing the rate of biochemical hypocalcemia and LOS for patients who undergo TT. Data were collected prospectively on a monthly basis and presented quarterly to a multidisciplinary team from Endocrinology, Pediatric Surgery, and Otolaryngology. PDSA cycles were used to make subsequent interventions and changes to the pathway with quarterly run charts developed to track the impact of interventions.

Outcome measures included rates of IV calcium gluconate use, rates of hypocalcemia, and LOS. Pathway adherence, defined by the appropriate pre-operative and post-operative labs being ordered and obtained, was the primary process measure analyzed. Initial interventions largely revolved around increasing pathway adherence. This project was reviewed by the Institutional Review Board of Phoenix Children's Hospital and determined to be a QI project and not human subjects research.

PDSA cycle 1: A run chart depicting interventions and adherence is presented in Figure 2. There were nine TT's performed during the first quarter of the project, with six instances meeting the definition of adherence. In addition to presenting this data to the multidisciplinary group, our first intervention was to send weekly emails containing the pathway to the on-call endocrine team. There were three TT's performed during the quarter after this intervention, with all three meeting the definition of adherence. A decrease in IV calcium gluconate was observed, however rates of biochemical hypocalcemia remained relatively unchanged.

PDSA cycle 2: Our second intervention was to start all patients on pre-operative calcium carbonate one week prior to surgery in an attempt to address rates of hypocalcemia. Three patients underwent TT in the quarter following this intervention, and all three developed biochemical hypocalcemia. Two patients met the definition of adherence. Feedback from the group suggested the creation of an order set (group of orders consisting of laboratories and medications) in our electronic health record (EHR) to assist the ordering provider. 
Total Thyroidectomy Calcium Management

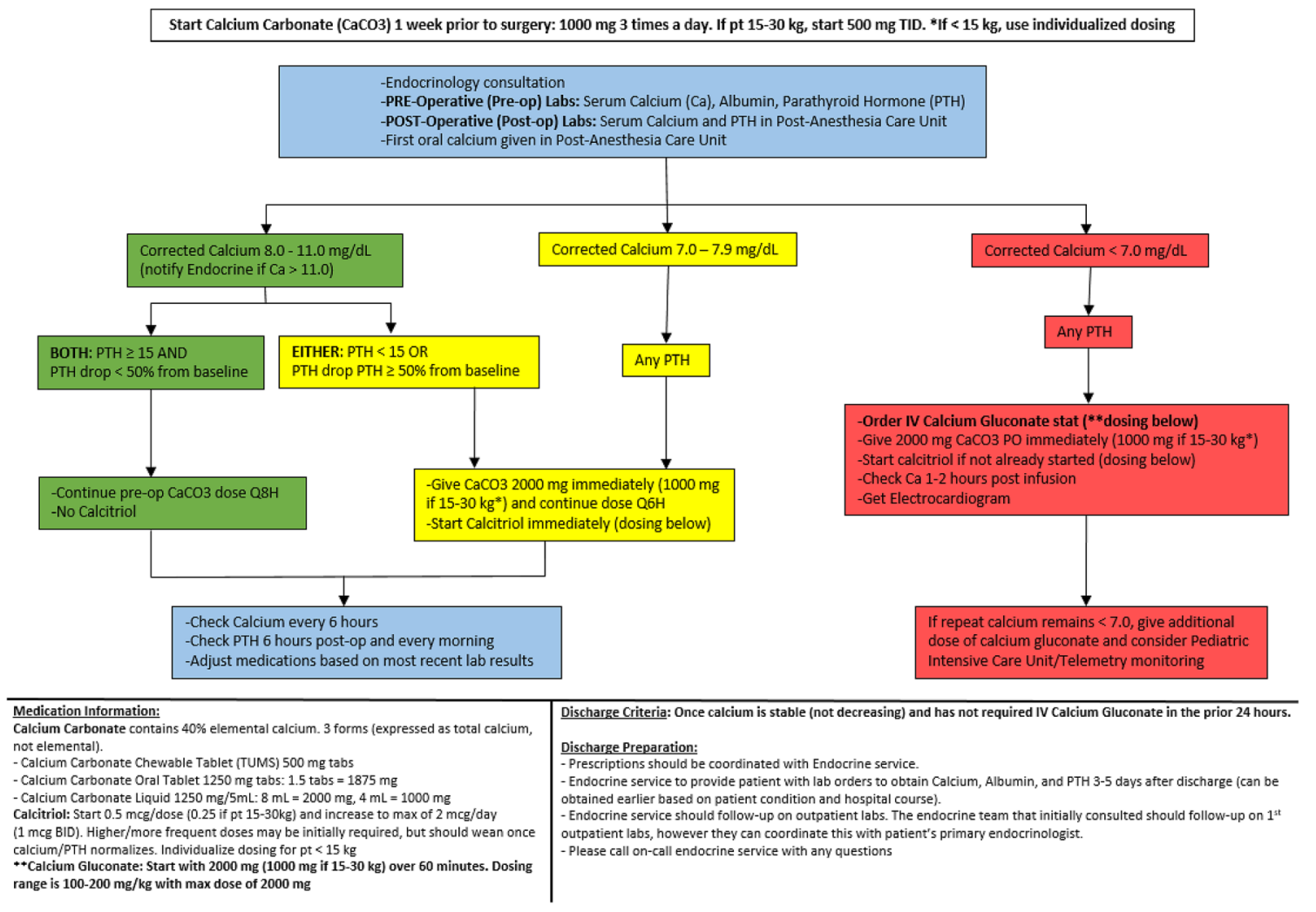

Figure 1: Clinical pathway for management of hypocalcemia after total thyroidectomy. All serum calcium values are expressed as $\mathrm{mg} / \mathrm{dL}$. Please refer to the "Materials and Methods" section of the text for a more detailed overview of the pathway.

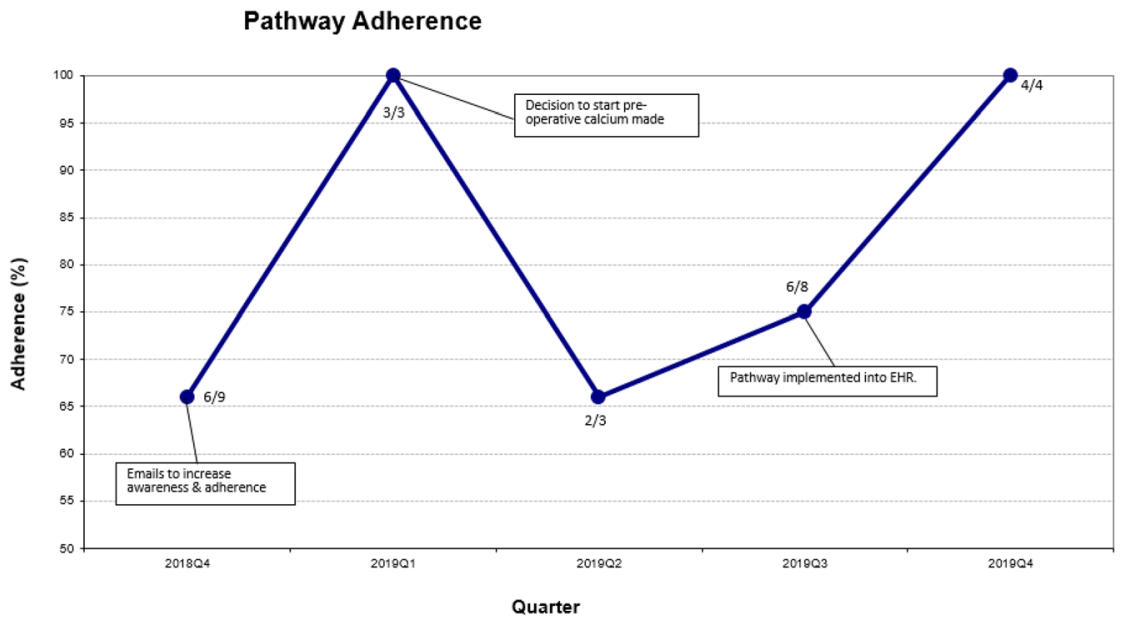

PDSA cycle 3: Our third intervention was the implementation of an EHR pathway order set to assist with ordering labs and medications. An additional eight TT's were performed during the three months it took to develop the order set, and adherence was $75 \%$ in that quarter. IV calcium gluconate use also increased. There were four TT's performed after EHR order set implementation, with 100\% adherence in this group. IV calcium gluconate use declined as well. There was no impact on biochemical hypocalcemia.

\section{Results}

Figure 2: Run chart for quarterly pathway adherence. Numerator is patients who met definition of adherence and denominator is total patients per quarter.

There were 27 total thyroidectomies performed in the 15 months since pathway implementation. Patient demographics are outlined in Table 1 . The mean age was $14.3 \pm 2.4$ years old. $89 \%$ of patients were female; $67 \%$ had Graves' disease and 41\% had thyroid cancer. One patient had 
Table 1: Demographics of 27 TT's performed since pathway implementation.

\begin{tabular}{lr}
\hline Patients & $\mathbf{n}(\%)$ \\
\hline Age (mean, SD) & $14.3 \pm 2.4$ \\
Female & $24(89)$ \\
Male & $3(11)$ \\
Graves disease & $17(63)^{\mathrm{a}}$ \\
Malignancy & $11(41)^{\mathrm{a}}$ \\
Neck dissection & $5(19)$ \\
\hline
\end{tabular}

Age reported in years. SD, standard deviation; TT, total thyroidectomy. ${ }^{\text {a}}$ One patient had both Graves' disease and malignancy as described in the text.

both Graves' disease and thyroid carcinoma. Of patients with malignancy, 3/11 (27\%) had central neck dissections, and 2/11 (18\%) underwent synchronous lateral neck dissections.

A run chart detailing interventions and IV calcium gluconate use is depicted in Figure 3. Thirteen patients (48\%) received IV calcium gluconate in the 15 months since pathway implementation. Twenty six patients $(96 \%)$ developed biochemical hypocalcemia over this time. Average LOS was $52.7 \mathrm{~h}$ (range 22.1-115.7) with a median LOS of $43.6 \mathrm{~h}$. All patients were started on calcitriol during hospitalization, and all patients were discharged home on both calcium carbonate and calcitriol.

In total, 21 out of 27 (78\%) TT's met the definition of adherence to the pathway. The most common reason for non-adherence was not obtaining pre-operative labs. Of the TT's that did not meet adherence, five out of six $(83 \%)$ received IV calcium gluconate. In contrast, eight out of 21 (38\%) that adhered to the pathway received IV calcium gluconate.

PTH levels of patients who underwent TT were also analyzed, including lowest post-operative PTH. Of the 13 patients who were administered IV calcium gluconate, 11 (85\%) had a PTH less than $15 \mathrm{pg} / \mathrm{mL}$ (considered low based on our lab's reference range), at some point during their hospitalization. The remaining two patients had lowest PTH levels of $29 \mathrm{pg} / \mathrm{mL}$ and $39.9 \mathrm{pg} / \mathrm{mL}$ respectively. There were 12 patients in which PTH nadir stayed above $15 \mathrm{pg} / \mathrm{mL}$, and only $2(17 \%)$ required IV calcium gluconate in that group.

One patient was readmitted with hypercalcemia; however this patient's care deviated from the designated pathway due to excessive calcium carbonate administration. There were no readmissions for hypocalcemia. There were no episodes of IV calcium gluconate extravasation.

\section{Discussion}

We set out to develop an institutional clinical pathway for the management of hypocalcemia after TT. This was done in the context of a QI project with the primary aim of decreasing IV calcium gluconate use from $68 \%$ to less than $40 \%$ over a span of 15 months. Secondary aims included decreasing biochemical hypocalcemia and LOS. After 15 months since implementation of our pathway, IV calcium gluconate use has decreased (though not to goal), but biochemical hypocalcemia and LOS remain relatively unchanged.

Prior to developing a clinical pathway, management of hypocalcemia after total thyroidectomy was variable at our institution. Standardization of management has impacted some measures, and provides an opportunity for robust outcome analysis. Initial challenges revolved around pathway adherence, which demonstrated the importance of buy-in from major stakeholders, an essential component of a successful QI project. Interventions including weekly emails, presenting project data to the multidisciplinary group, and implementing the pathway into the EHR were aimed at increasing pathway awareness and adherence. As buy-in from the practitioners overseeing patients and placing orders increased, so did adherence.

As pathway adherence increased, we observed a decrease in IV calcium gluconate use. While pathway

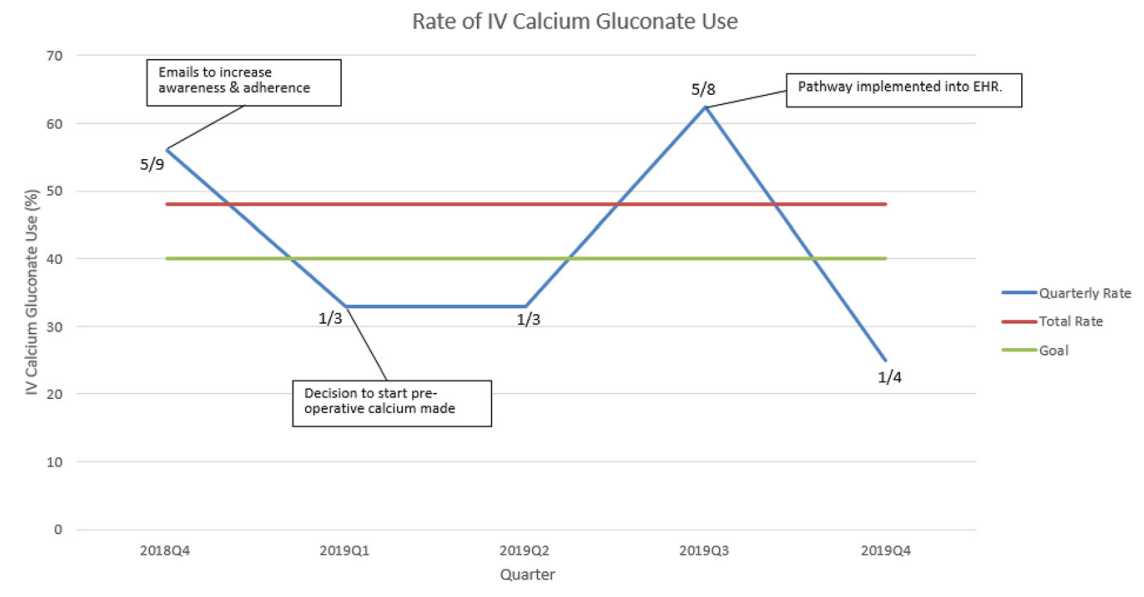

Figure 3: Run chart for IV calcium gluconate. Numerator is patients received IV calcium gluconate and denominator is total patients per quarter. The red line represents the total rate $(48 \%)$ for the current duration of the project while the green line represents our goal rate of $40 \%$. 
adherence may have played a role in reducing IV calcium gluconate use, there continued to be instances in which IV calcium gluconate was required as directed by the pathway itself. This observation suggests that further interventions and pathway revisions are likely needed and also that other factors likely influence the need for IV calcium gluconate.

The primary risk factor for needing IV calcium gluconate in our patient population was post-operative hypoparathyroidism and PTH level. This is consistent with the physiology of calcium homeostasis. PTH increases serum calcium both directly at the level of the kidney and bone, and indirectly by stimulating the enzyme $1 \alpha$-hydroxylase. This enzyme in the kidney converts inactive 25-hydroxyvitamin D to active 1,25-dihydroxyvitamin D, also known as calcitriol. Calcitriol subsequently increases calcium and phosphorous absorption from the gut, with PTH secretion creating an overall net effect of increased serum calcium. Low post-operative PTH levels have been associated with hypocalcemia in pediatric studies $[5,10]$.

Freire et al. suggested the utility of PTH levels in predicting post-operative hypocalcemia in children using a longitudinal cohort study of 32 pediatric patients undergoing TT by a single surgeon [10]. These data were used to develop an algorithm to evaluate risk of hypocalcemia based on PTH levels 5- and 60-min after thyroid removal. A more recent retrospective study of 38 patients assessing the utility of intact PTH levels in managing hypocalcemia after TT in children showed a cut-off PTH level of less than $26 \mathrm{pg} / \mathrm{mL}$ had $100 \%$ specificity and $75 \%$ sensitivity in predicting hypocalcemia, though it also concluded that PTH level alone may not be sufficient to predict postoperative normocalcemia [5].

The administration of calcitriol is meant to offset the hypoparathyroidism and decreased production of intrinsic calcitriol that often results after TT. Since pathway implementation, all patients were started on calcitriol at some point during their hospitalization. Despite initiation of calcitriol often within a few hours after completion of surgery, many patients still developed severe hypocalcemia requiring IV calcium gluconate. While oral calcitriol is rapidly absorbed, it takes several doses to achieve steady state and reach maximum effect. As a result, our patients may not be getting calcitriol medication early enough to prevent severe hypocalcemia.

This conclusion is further supported by a recent study from Baumgarten et al. that reported institutional complication rates after TT in addition to detailing clinical practice guidelines stratifying risk with intraoperative parathyroid hormone (ioPTH) measurements and early intervention with IV calcitriol [1]. The ioPTH is measured at the initiation of surgery, 10-, and 30-min after thyroid removal. If the second or third PTH is $<10 \mathrm{pg} / \mathrm{mL}$, IV calcitriol is administered in the operative room or recovery room. In the four years reported since instituting this strategy, none of the 270 patients undergoing TT required rescue IV calcium. We are currently considering the logistics of ioPTH and either pre-operative or intraoperative IV calcitriol use as possible future interventions for our pathway.

Undertaking this process as a QI project provided an opportunity to develop a pathway tailored to the local context. What works at one institution may not be possible at another, however if an institution is able to standardize care, the pathway can subsequently and continuously be adjusted to enhance outcomes. QI principles have been used to develop clinical pathways for a variety of different conditions within major children's hospitals [11] and it stands to reason that the post-operative management of TT can benefit from the same process.

There are several limitations to this project. Given the nature of chart review, it was not always possible to discern the true indication for IV calcium gluconate administration, and there may have been cases in which it was needed due to post-operative nausea and vomiting and inability to tolerate calcium and/or calcitriol taken orally. Additionally, this is a single center experience, and though we are a relatively high volume center, the number of thyroidectomies varied over time. The involvement of different surgeons also introduces inherent variability which may influence outcomes. In our current state, it is not feasible to have a single surgeon perform this procedure, nor is it logistically possible to transfer all patients requiring TT out of state to a higher-volume center. We have taken steps to address this variability, including limiting the number of surgeons who perform TT in order to optimize surgeon volumes. In addition, Vitamin D levels were not routinely measured on all patients. As vitamin D deficiency can reduce the absorption of oral calcium, it is possible that this may have influenced our results. We are currently taking steps to more uniformly measure and optimize vitamin D status in the weeks prior to surgery.

In summary, we were better able to understand management and patient outcomes for TT at our institution by implementing a clinical pathway in the context of a QI project. IV calcium gluconate use decreased from 68 to $48 \%$ over a span of 15 months. Rates of hypocalcemia and LOS remained relatively unchanged. This process confirmed what was previously known about the importance of post-operative PTH level with respect to the risk of developing hypocalcemia. As a result, we are now considering adding pre-operative or intraoperative calcitriol as a future intervention. 
We propose that many of our limitations, specifically sample size and variety of surgeons, are real-world challenges that other children's hospitals face with regard to this procedure, and offer that the lessons learned and the advantages of implementing a clinical pathway like this as a QI project are more widely applicable. This remains an on-going QI project with the ultimate goal of producing a pathway that best utilizes our institution's specific infrastructure and resources to make a more significant clinical impact on IV calcium gluconate use, hypocalcemia, and LOS.

Research funding: None declared.

Author contributions: All the authors have accepted responsibility for the entire content of this submitted manuscript and approved submission.

Competing interests: The funding organization(s) played no role in the study design; in the collection, analysis, and interpretation of data; in the writing of the report; or in the decision to submit the report for publication.

Informed consent: Informed consent was obtained from all individuals included in this study.

\section{References}

1. Baumgarten HD, Bauer AJ, Isaza A, Mostoufi-Moab S, Kazahaya K, Adzick NS. Surgical management of pediatric thyroid disease: complication rates after thyroidectomy at the Children's Hospital of Philadelphia high-volume Pediatric Thyroid Center. J Pediatr Surg 2019; 54: 1969-75.
2. Kundel J, Thompson G, Richards M, Qiu L, Xiu L, Cai Y, et al. Pediatric endocrine surgery: a 20-year experience at the Mayo clinic. J Clin Endocrinol Metabol 2014; 99: 399-406.

3. Al-Qurayshi Z, Hauch A, Srivastav S, Aslam R, Friedlander P, Kandil E. A national perspective of the risk, presentation, and outcomes of pediatric thyroid cancer. JAMA Otolaryngol Head Neck Surg 2016; 142: 472-8.

4. Patel NA, Bly RA, Adams S, Carlin K, Parikh SR, Dahl JP, et al. A clinical pathway for the postoperative management of hypocalcemia after pediatric thyroidectomy reduces blood draws. Int J Pediatr Otorhinolaryngol 2018; 105: 132-7.

5. Jiang W, Lee E, Newfield RS. The utility of intact parathyroid hormone level in managing hypocalcemia after thyroidectomy in children. Int J Pediatr Otorhinolaryngol 2019; 125: 153-8.

6. Yu YR, Fallon SC, Carpenter JL, Athanassaki I, Brandt ML, Wesson $\mathrm{DE}$, et al. Perioperative determinants of transient hypocalcemia after pediatric total thyroidectomy. J Pediatr Surg 2017; 52: 684-8.

7. Chen Y, Masiakos PT, Gaz RD, Hodin RA, Parangi S, Randolph GW, et al. Pediatric thyroidectomy in a high volume thyroid surgery center: risk factors for postoperative hypocalcemia. J Pediatr Surg 2015; 50: 1316-9.

8. Agac AA, Kutun S, Cetin A. Are the characteristics of thyroid cancer different in young patients? J Pediatr Endocrinol Metab 2014; 27: 497-502.

9. Noordzij JP, Lee SL, Bernet VJ, Payne RJ, Cohen SM, McLeod IK, et al. Early prediction of hypocalcemia after thyroidectomy using parathyroid hormone: an analysis of pooled individual patient data from nine observational studies. J Am Coll Surg 2007; 205: 748-54.

10. Freire AV, Ropelato MG, Ballerini MG, Acha O, Bergadá I, de Papendieck LG, et al. Predicting hypocalcemia after thyroidectomy in children. Surgery 2014; 156: 130-6.

11. Lavelle J, Schast A, Keren R. Standardizing care processes and improving quality using pathways and continuous quality improvement. Curr Treat Options Pediatr 2015; 1: 347-58. 